\title{
Expression of cysteine proteinases and cystatins in parasites and use of cysteine proteinase inhibitors in parasitic diseases. Part II: Arthropods
}

\author{
Sherif M Abaza
}

Medical Parasitology Department, Faculty of Medicine, Suez Canal Uinversity, Ismailia, Egypt

\begin{abstract}
Most of the cysteine proteases (CPs) expressed by ectoparasites belong to the papain-like superfamily (clan CA, family C1) and are associated with the physiological events during different developmental life stages. Cathepsins, legumains/asparaginyl endopeptidases and caspases (clan CA, family C1, C13 and C14, respectively) play a major role in mosquito biology. There is accumulating evidence that CPs expressed by arthropods may play a role in clinical presentations of some transmitted parasitic disease, e.g. babesiosis. During blood meals, salivary glands secrete bioactive substances, including CPs, to exert several pharmacological properties that assist ectoparasites to evade host defense responses and to induce inflammatory reactions. Furthermore, they play an important role in extracellular and intracellular proteins degradation as well as their processing. In this regard, cystatins (CYSs) were found to inhibit insect CPs suggesting its use as targets for control of mites and ticks. The present review aims to identify different CPs expressed in ectoparasites, the main vectors of several endemic diseases such as malaria, babesiosis, Chagas' and Lyme disease. Meanwhile, it highlights different CYSs that could be used as biological insecticides to control diseases transmitted by mites and ticks.
\end{abstract}

Keywords: arthropods, cathepsin, cystatins, vaccine, vector control.

Received: 7 November, 2018, Accepted: 8 December, 2018.

Corresponding Author: Sherif M Abaza, Tel.: 01005243428, E-mail: smabaza@hotmail.com

Print ISSN: 1687-7942, Online ISSN: 2090-2646, Vol. 11, No. 3, December, 2018.

\section{Abbreviations}

AEP: Asparaginyl endopeptidase; BFA: Blood feeding arthropod; Cath: Cathepsin; CP: Cysteine proteinase; CPI: Cysteine proteinase inhibitor; CYS: Cystatin; RNAi: RNA interference method for gene knock out.

\section{Mosquitoes}

Ectoparasites, especially blood feeding arthropods (BFAs), possess salivary glands secreting bioactive substances, among which are cysteine proteinases (CPs) that allow evasion of host response and the production of inflammatory reactions ${ }^{(1)}$. In addition, the secreted CPs assist in hemoglobin digestion during blood meals essential for egg production. It was shown that E64, a general cysteine proteinase inhibitor (CPI) was used to reduce fecundity of $C$. pipiens females and results revealed the presence of an enzyme band, most probably that of a CP, in untreated mosquitoes ${ }^{(2)}$.

Cathepsin-B-like (Cath-B-like) was characterized from yellow fever and malaria transmitting mosquitoes, and named as vitellogenic cath B (VCB). It degrades vitellogenin, the major yolk protein precursor $^{(3)}$. In 2001, examination of CPs stored in the ovaries of Culex pipiens showed that Cath B- and L-like have a major role in development and maturation of Culex ovaries. It was shown that both Caths facilitate degeneration of developing follicles (follicular atresia) or oocysts resorption (oosorption) ${ }^{(4)}$. The investigators suggested that follicular atresia and oosorption should occur to maintain the balance between number of eggs production and physiological as well as environmental factors such as insufficient blood meals, absence of males, lower temperatures, and lack of suitable oviposition sites. Three years later, the same investigators confirmed the effects of ovarian CPs using electron microscopy. Results revealed gradual degradation of the internal structures including yolk granules in the oocyte with appearance of irregularly shaped epithelial cells and signs of apoptotic cells ${ }^{(5)}$.

In a review article published in 2006, the reviewers discussed self-defense mechanism(s) involved in Plasmodium-infected mosquitoes to repair invaded mid-gut epithelium and to balance mosquitoes' survival. Death of Plasmodium ookinetes in infected mosquitoes was associated with caspase-like activity. Although it is homologous to mammalian caspases, it was not detected in Plasmodium genome, suggesting the role of other mosquitoes CPs, e.g. Caths, in repair and survival ${ }^{(6)}$. Based on a recent study conducted in Brazil( ${ }^{(7)}$ on Culex quinquefasciatus, the investigators identified the activity of Cath B in egg extracts $24 \mathrm{~h}$ after oviposition. It was shown that Cath B significantly decreased in fertilized eggs compared to extracts from vitellogenic ovaries or unfertilized eggs, suggesting its role in yolk 
degradation during embryogenesis. This result was confirmed using a specific Cath B inhibitor, C-074, but not with other tested CPIs in extracts of unfertilized eggs. In addition, the transcriptional profile of two Cath $B$ genes was determined, and their enzymatic activities were expressed simultaneously in the vitellogenic females. Based on the data obtained from VectorBase (vectorbase.org), the investigators found significant similarity between the transcriptional profiles of both Caths and vitellogenin, the primary source of amino acids and lipids for embryonic development. Accordingly, the investigators hypothesized that Cath B cooperate in vitellin degradation during embryogenesis.

A novel caspase (AeDredd) was identified and characterized from all developmental stages of Aedes aegypti with highest expression in early pupae. It proved to have an essential role of apoptosis in the innate immune response of vectors towards intracellular parasites such as viruses ${ }^{(8)}$.

\section{Tsetse fly}

In an attempt to investigate gut enzymes involved in digestion of dietary proteins in Glossina morsitans, the vector of African trypanosomiasis, Yan et al., ${ }^{(9)}$ molecularly characterized the gene encoding Cath B (GmCatB). They also characterized two other genes encoding zinc-metalloprotease and zinccarboxypeptidase. Gene sequence analysis of GmCatB reveled 254 amino acids with a molecular weight of $29 \mathrm{kDa}$. Later, recombinant GmCatB was found to produce the highest proteolytic activity at $\mathrm{pH} 4.0$. It degraded bovine hemoglobin and serum albumin, and was inhibited by E-64, i.e. it belongs to papain-like CP family ${ }^{(10)}$

\section{Bloodsucking bug (Triatoma spp.)}

Cath D as well as Cath B- and Cath L-like activities were identified in the gut extracts of Triatoma spp. ${ }^{(11,12)}$. In addition, Cath-like enzymes were also involved in the proteolytic activation of canatoxin that results in production of entomotoxic peptide(s) involved in the deleterious effects in Chagas disease ${ }^{(13)}$.

\section{Mites}

Several studies were conducted to evaluate the efficacy of CPIs and/or CYS A in treatment of allergic syndromes caused by house dust mites (HDMs), Dermatophagoides species. Sequence analyses of allergens (Der $p 1$ and $\operatorname{Der} f 1$ ) expressed by $D$. pteronyssinus and $D$. farina, the most common HDMs causing allergic asthma and rhinitis as well as atopic dermatitis, showed similarity with that of $\mathrm{CPS}^{(14)}$. Group 1 allergen (Der-1) is a glycoprotein which exhibits CP activity as determined by comparative modeling with papain, actinidin and papaya peptidases (clan CA, family $\mathrm{C} 1)^{(15)}$.
In atopic dermatitis, Der-1 was found to have major roles in cleavage of human CD23 and CD25, enhancement of total and specific IgE production, inactivation of the endogenous human cystatins (CYSs), and stimulation of human keratinocytes to produce IL-8 involved in the pathogenesis of atopic dermatitis ${ }^{(16-18)}$. The results of a study conducted in UK revealed the significant potency of PTL11028 as a selective irreversible inhibitor of CP activity of Der-1 ${ }^{(19)}$. On the other hand, CYS A is a skin-derived dominant inhibitor against proteolytic activity of major HDMs allergens. Cystatin A (CYS A) also was used to inhibit keratinocytes stimulation as well as IL-8 production ${ }^{(20)}$. One year later, Ogawa et al.,(21) reported that Der-1stimulated keratinocytes caused upregulation of the release of granulocyte-macrophage colony-stimulating factor which contributes in the pathogenesis of atopic dermatitis. The investigators showed that CYS A had a major homeostatic role against atopic dermatitis.

In respiratory asthmatic attacks caused by HDMs, Der-1 was found to target multiple proteins involved in the control of IgE synthesis and production ${ }^{(22)}$, degradation of endogenous protease inhibitors ${ }^{(23)}$, and surfactant proteins ${ }^{(24)}$, increasing the contact between allergens and dendritic cells (antigen presenting cells) beneath the bronchial epithelial barrier. To discover new drugs for treatment of asthma caused by HDMs, a study was conducted in 2014. The investigators described the mechanisms by which HDMs Der-1 allergens promote allergic sensitization in asthma; cleavage of the epithelial tight junctions by their proteolytic activities followed by release of chemokines and other mediators (e.g., IL-13, IL-33 and IL-25). In their study, the investigators designed a proposal to identify reversible Der-1 inhibitors and claimed that using this generated program might lead to emergence of promising candidates for treatment of allergic asthma caused by HDMs ${ }^{(25)}$. Recently, it was shown that Der-1 activates human receptor MRGPRX1 enhancing IL-6 production which contributes in pathogenesis of allergic rhinitis and allergic asthma ${ }^{(26)}$.

Degradome sequencing, a method used to provide a comprehensive analysis of RNA degradation and to identify micro-RNA cleavage sites, showed that Der-1 accounts for $50 \%$ of protease transcription and $22 \%$ of the total protease transcripts ${ }^{(27)}$. Two years later, Randall and his colleagues ${ }^{(28)}$ conducted a comparative analysis of proteases from $D$. farinae and found that Der-1 showed high simultaneous expression as well as high stability than other proteases in $D$. farinae. In addition, they detected two other allergens (Der-3 and Der-6) and two metallo-proteases with similar stability and expression. Therefore, it was recommended to characterize the detected proteases and to determine if they can stimulate human IgE production.

Regarding scabies' Sarcopetes spp. mites, Australian investigators discovered a unique multi-copy family of 
genes encoding CPs with their catalytic sites inactivated by mutation. As they are absent in non-burrowing, free living mites, the investigators postulated that these encoding genes are evolved to adapt Sarcopetes spp. to the parasitic lifestyle to invade host tissues, and they were named SMIPP-Cs (scabies mite inactivated cysteine protease paralogs). Five SMIPP-Cs homologs were identified (a-e). Sequence gene analysis, prior and after activation, revealed replacement of glutamine (homologs a and b), and leucine (homologs c-e), by active histidine ${ }^{(29)}$. Two years earlier (2002), another group of British investigators detected close similarity between S. scabiei CPs and HDMs Der-1 allergens. Interestingly, genomic analysis of two Dermatophagoides spp. and sheep scab mite, Psoroptes ovis showed closed relatives in a single encoding gene $\mathrm{e}^{(30)}$.

In order to elucidate SMIPP-Cs function(s) of Sarcopetes spp. in different hosts with relation to its host specificity, Fernando et al., ${ }^{(31)}$ bioinformatically analyzed SMIPP-Cs from human, pig and dog mites. The investigators detected five activated homologs in the three Sarcopetes spp. of medical and veterinary importance. Although SMIPP-Cf was detected only in mites invading pigs and dogs, human mites showed two different versions of SMIPP-Cc with absence of SMIPPCf. Accordingly, it was postulated that homolog's amplification and sequence variation occurred prior and after host adaptation, respectively. Amino acid sequences of genes encoding CPs are conserved among each other and phylogenetic alignment analysis with previous studies suggested that they belong to monophyletic gene family. In addition, the investigators detected SMIPP-Cs expression in all burrowing stages with the highest in female mites. Localization studies using recombinant antibodies showed constant intense localization in the intestinal tract and feces indicating their gut origin. Although the investigators assigned proteolytic activity related to digestion and providing nutrients as well as inhibition of host complement activation and evasion of host immune response to serine proteases, they recommended further studies to assign the definite role of SMIPP-Cs in interaction with host epidermal tissue and host immune response ${ }^{(31)}$.

\section{Ticks}

In a report published in 2011, the reviewers summarized the differences between soft and hard ticks as obligate blood feeders: 1) nymphs and adults of both sexes of soft ticks feed rapidly and several times during their life stages while hard ticks take a single large blood meal during their whole life cycle; 2) soft ticks feed within $30-60 \mathrm{~min}$, while the process is slow for 6-9 days in hard ticks; 3) feeding and oviposition are repeated, lasting for 150-200 days and are not related to mating in soft ticks, while only mated female hard ticks die after oviposition of a single large batch of eggs. The reviewers also discussed all CPs expressed in several species of soft and hard ticks. In addition, they claimed that the feeding process involving degradation of host hemoglobin is a complex process that requires involvement of several gut associated CPs as well as aspartic proteases. Beside hemoglobin degradation, they discussed the major role played by CPs in embryogenesis with special emphasis on degradation of yolk proteins, mainly vitellin. In that issue, they claimed that the cascade or pathway employed in ticks is more conserved among ticks compared to other $\mathrm{BFAs}^{(1)}$.

Two other reviews were published to express the importance of tick CYSs in development of either a vaccine candidate for vector control instead of acaricidal compounds, or novel drug target to treat human diseases transmitted by ticks. In the first review ${ }^{(32)}$, overall 21 tick CYSs were reviewed and only nine of them were functionally characterized in the reviewed literature. Interestingly, up to that date (2012), all CYSs were identified as potent inhibitors of the proteolytic activity of papain-like CPs (Caths), but not legumains (asparaginyl endopeptidase; AEP) due to lack of binding site in their protein sequence, e.g. legumains from Ixodes ricinus (IrAE) and Haemaphysalis longicornis (Hllgm1 and 2). The reviewers postulated this observation to the involvement of these CPs in tick physiology rather than blood feeding process. The identified CYSs were categorized according to their types. Type I (stefins) were assigned to Rhipicephalus haemaphysaloides (RhCys-1), H. longicornis (HilCys-1), and Dermacentor variabilis (DvM602). On the other hand, type II CYSs (sialostatins) were identified in I. scapularis (IsSL and IsSL-2), H. longicornis (HilCys 2 and 3), Ornithodoros moubata (OmC 1 and 2), Amblyomma americanum (AaSL), D. variabilis (DvM226 and DvM334), and $R$. haemaphysaloides (RhCys-2). In the second review ${ }^{(33)}$, it was claimed that $~ 85 \%$ of the identified transcripts of the detected tick CYSs belonged to the extracellular group, suggesting predominant immunomodulatory role.

Concerning the use of CPIs of natural herbs, amaranth CYS proved to have effective proteolytic activity against a wide range of CPs detected in ticks infestation of plants, suggesting its role in control of insect pests ${ }^{(34)}$. The present review will only focus on the role and function(s) of CPs and CYSs, of medically important ticks which transmit or potentially transmit human diseases.

Degradation of host proteins in tick gut cells is a complex process that is not performed only by hemoglobinase enzyme activity. The digestive network consists of AEP (clan CD, family C13) and Caths B, $\mathrm{C}$ and L (clan CA, family $\mathrm{C} 1$ ) as well as gut-related aspartic and leucine aminopeptidases ${ }^{(35)}$. Accordingly, the overall hemoglobinolytic activity measured in tick gut tissue extracts during feeding was highly elevated from the $6^{\text {th }}$ day after attachment to the host, which corresponds to expression of these $\mathrm{CPs}^{(36)}$. From 
clan CD, AEP was the first CP identified, localized, characterized and fully sequenced in I. ricinus; the major European hard tick species which transmits Lyme disease and tick-borne encephalitis. It was observed that IrAEP was exported from the lysosomal vesicles, and extracellularly localized within the peritrophic matrix of engorged females at the $5^{\text {th }}$ day of feeding. Its proteolytic activity on host hemoglobin degradation was effectively inhibited with a legumainspecific inhibitor. The investigators discussed their results and claimed that hemoglobin degradation was strongly similar to that observed in the regurgitates of $S$. mansoni adult worms ${ }^{(36)}$. One year later, the same investigators confirmed the AEP role in hemoglobin degradation, in contrast to other BFAs which rely on serine peptidases ${ }^{(37)}$.

Another report characterized cDNA of the genes encoding AEP from H. longicornis (Hllgm and Hllgm2), a vector incriminated in transmission of babesiosis. Endogenous Hllgm was immunolocalized in the midgut epithelium of all developmental stages with a $38 \mathrm{kD}$ molecular weight. In addition, the investigators found that its potent proteolytic activity on both bovine hemoglobin and serum albumin was inhibited by the thiol blocking reagents, suggesting its major role in blood meal process ${ }^{(38)}$. To elucidate legumains functions in $H$. longicornis, the same investigators utilized RNA interference (RNAi) techniques for gene knock down. Results showed significant damage of the midgut tissues with reduction in tick body weight. Moreover, there was significant delayed onset of oviposition with reduced egg number and increased structurally deformed eggs. Therefore, the investigators concluded the use of specific legumains inhibitors as vector control strategy to control diseases transmitted by $H$. longicornis ${ }^{(39)}$. In another trial for tick population control, a group of American scientists conducted transcriptomic studies on expressed sequence tag (ESTs) from cDNA libraries from female tick midguts at varying stages of feeding to identify transcripts involved in blood meal digestion in $D$. variabilis. It was observed that $\sim 25 \%$ (19 out of 82 ESTs) were expressed in the $6^{\text {th }}$ day fed midguts. Moreover, phylogenetic analysis with significant sequence similarity to the reported CPs in other ticks revealed presence of three major groups of CPs; legumain, Caths B and L. The investigators recommended further studies to investigate the role of these peptidases in development of new strategy in tick control $^{(35)}$

Endogenous longipain (known later as Cath B) was immunolocalized at lysosomal vacuoles of the midgut epithelium of $H$. longicornis. Utilizing gene knock down techniques, the investigators showed that ticks lacking endogenous Cath B significantly increased the number of Babesia parasites both in vitro and in vivo, compared to control, through specific adherence to the parasite membranes. Accordingly, the investigators hypothesized that Cath B showed pivotal role in $H$. longicornis survival, host hemoglobin degradation and as defense response against Babesia merozoites. Therefore, they recommended further studies to elucidate the structural features of endogenous Cath $\mathrm{B}$ of $\mathrm{H}$. longicornis to be used to control babesiosis transmission in ticks ${ }^{(40)}$. Another CP localized in the midgut of $H$. longicornis was molecularly characterized and termed HICPL-A due to its sequence similarity to Cath L. The HICPL-A gene expression was up-regulated by the blood-feeding process. Its proteolytic activity in degrading bovine hemoglobin was inhibited by three CPIs including E-64 ${ }^{(41)}$.

Another important role played by CPs in ticks is embryogenesis, specifically the degradation of yolk proteins. In that way, CPs might help tick survival by providing amino acids for protein catabolism enabling larval survival until the first blood meal is achieved. In fact, vertebrate blood provides an essential source of nutrients for energy metabolism to support demanding activities including embryogenesis ${ }^{(42)}$.

Salivary gland transcriptomic studies were conducted in unfed and fed $R$. haemaphysaloides which is a common ectoparasite in cattle, horse, sheep, and dogs, and the primary vector of bovine babesiosis as well as potential vector of human Kyasanur forest disease (Asian viral hemorrhagic fever). In their publication, sialo-transcriptome was analyzed, and the expressed CPs were confirmed by real-time PCR in salivary glands of different developmental stages during blood feeding. Results revealed successful cloning of four genes encoding expression of Caths B and $\mathrm{L}$, and caspases 1 and 8 as well as autophagy-related genes (ATGs). As Caths and caspases were expressed at early and late phases of tick engorgement, respectively, the investigators suggested assignment of hemoglobin degradation and inhibition of host cytokines for the role and function(s) of the identified CPs, respectively. They also recommended further studies to characterize these CPs which may help in development of novel drug targets and/or a vaccine candidate for vector control ${ }^{(43)}$.

On the other hand, autophagy is another pathway leading to apoptosis, with activity involvement of CPs, but controlled by ATG4 and ATG8. Recently, Yu et al. ${ }^{(44)}$ succeeded to identify two ATG8s. It is well known that caspase 1 plays a major role in salivary gland apoptosis of $R$. haemaphysaloides 3-4 days after tick attachment. Although the investigators performed silencing RNAi technique to knock out the genes encoding caspases 1 and 8, they still observed apoptosis, but delayed. Accordingly, the investigators suggested that salivary gland apoptosis of $R$. haemaphysaloides is affected by both pathways; genes encoding caspases and ATGs ${ }^{(44)}$.

During the period 2002-2015, a group of scientists working in National Institute of Health $(\mathrm{NIH})$ published several reports ${ }^{(35,45-51)}$. In addition, Ribeiro and Franischetti ${ }^{(52)}$ reviewed strategies involved in 
hard ticks during blood feeding. On year earlier, they conducted transcriptome studies for saliva components of the black-legged tick (I. scapularis), the main USA vector of Lyme disease. They showed the presence of sialome protein, and its sequence analysis revealed homologous similarity with several protease inhibitors with different conserved cysteine residues ${ }^{(45)}$. In 2006, Kotsyfakis and his colleagues ${ }^{(46)}$ named it sialostatin-L (ISSL) because of its significant inhibitory proteolytic activity against human Cath $\mathrm{L}$, and to a lesser extent Cath C. In addition, their results showed that ISSL targeted several essential enzymes required for both in vitro and in vivo proliferation of cytotoxic T lymphocyte (CTL) in cell line and mouse model, respectively. The investigators attributed the significant reduction of extra- and intracellular compartments to IsSL potent anti-hemostatic and anti-inflammatory activities. One year later, the same investigators discovered two genes encoding SL involved in proteolytic actions against host papain-like CPs during blood meals, suggesting expression of two CYSs. In addition, they observed an important difference between their transcript abundance during blood meals, as SL is required to reduce proliferation at the bite site, SL2 accumulates to perform its actions later as observed by increased transcription. When the investigators compared the actions of both SLs in hard and soft ticks (Ornithodoros moubata), they observed that those of the latter (rapid BFA) perform their main role in the midgut rather than in the salivary gland. Accordingly, the investigators hypothesized the immunosuppressive activity of SL2 during the prolonged blood meal. To confirm their hypothesis, the investigators utilized reverse genetic approach employing RNAi to knock out both genes, and they observed increased numbers of dead ticks due to strong host primary immune response. Meanwhile, ticks' rejection was observed in subsequent booster infestation. Therefore, the investigators concluded that development of inhibitor(s) against both SLs could be valuable vaccine against Lyme disease ${ }^{(47)}$.

To elucidate the immunosuppressive effect(s) of IsSL2, two further studies were conducted in 2015 in which the investigators demonstrated its mechanisms of action to inhibit interferon (IFN) signaling in host dendritic cells. Their results postulated three mechanism; interference with IFN-triggered signal transduction through decreasing phosphorylation process, inhibition of IFN- $\beta$-mediated induction of IFNsensitive genes in stimulated-dendritic cells and in vitro promotion of virus replication in dendritic cells ${ }^{(48,49)}$. However, other investigators from Czech Republic also observed the efficacy of IsSL2 as an immunosuppressive CYS in treatment of experimental asthma through its potent impairment of IL-9 production by Th9 cells and reduced expression of other asthmatic factors in mast cells. It was found that experimental application of IsSL2 in asthmatic mice (challenged with OVA antigen) significantly blocked eosinophilia and airway hyperresponsiveness ${ }^{(53)}$.
For A. americanum, a common USA vector for several human diseases and known also as turkey tick, another SL (AaSL) detected in the salivary glands and midgut was partially sequenced due to design error of the cloning primers based on the SL gene, however, its partial sequence was $\sim 100 \%$ identical to IsSL. When AaSL was investigated for its immunomodulatory role, it showed significant inhibition of immune response(s), suggesting capability of $A$. americanum utilizing its secretory AaSL to evade host immune response through disruption of normal antigen processing in host antigenpresenting cells. It was shown that knock out of the gene encoding $A a S L$ led to 90 and 50\% reduction in transcript abundance in the early and late phases of feeding, respectively, as well as decreased tick body weight, detachment of $35 \%$ ticks after 1 day and over $50 \%$ mortality of attached ticks, compared to control ticks ${ }^{(50)}$.

It is worth mentioning that a single CYS type 1 (DvM602) and two of type II (DvM334 and DvM226) were identified in $D$. variabilis and showed high amino acid sequence similarity to the other described CYSs. However, their inhibitory functions were not determined ${ }^{(35)}$. Another group of investigators from Czech Republic also, described two CYSs (OmC1 an 2) in soft ticks, O. moubata, the vector transmitting African swine fever virus and African tick-born relapsing fever. While $O m C 1$ transcripts were found only in the midguts of unfed ticks, $\mathrm{OmC} 2$ transcripts were found in all tissues and both possessed inhibitory proteolytic activity against Caths $\mathrm{B}, \mathrm{C}$, and $\mathrm{H}$ as well as papain ${ }^{(54)}$. In 2010, the crystal structure of $\mathrm{OmC} 2$ was characterized, and the investigators found its efficient inhibition of the proteolytic activity of endogenous (tick) Caths L and $\mathrm{S}$ as well as exogenous (host) Caths $\mathrm{B}, \mathrm{C}$ and $\mathrm{H}^{(51)}$. Both in vitro and in vivo studies were conducted to demonstrate its significant role in reduction of proinflammatory cytokines (TNF- $\alpha$ and IL-12), and antigenspecific CD4+ T cells. Accordingly, the investigators hypothesized that $O m \mathrm{C} 2$ is capable of suppressing host recognition of salivary antigens (endogenous Caths $\mathrm{L}$ and S) to manipulate the adaptive immunity which efficiently facilitates repeated blood meals on the same host. Meanwhile its ability to inhibit host Caths B, C and $\mathrm{H}, \mathrm{OmC2}$ contributes to decrease host innate immunity. Therefore, a vaccination experiment utilizing OmC2 antibodies was performed and showed significant increase in mortality rate of 0 . moubata nymphs after blood meals from immunized animals. The investigators concluded its usefulness as a candidate vaccine to control disease transmission ${ }^{(51)}$.

Apart from the NIH publications, three CYSs were molecularly characterized, and identified in salivary content of H. longicornis. The identified CYSs (HlCys 1-3) showed efficient inhibition of the proteolytic activity of papain and Cath $\mathrm{L}$, but not to Cath $\mathrm{B}$. Their endogenous nature was immunolocalized in the acini of the salivary gland of adult ticks, their expressed transcripts were highly up-regulated in the early blood feeding processes, 
and their genes were sequenced ${ }^{(55-57)}$. Moreover, another two CYSs were isolated from $R$. haemaphysaloides (RhCys-1 and 2) and cloned to investigate their inhibitory activities against different Caths. To assess their role and function(s), expression analyses in different developmental stages and RNAi studies were conducted. Results revealed their efficient inhibitory activity against all tested Caths, with strongest affinity to Cath $\mathrm{S}$, high expression in egg stage, and essential role in embryogenesis ${ }^{(58,59)}$.

Recently, in vitro and in vivo effects of RhCyst- 1 in four tumor cell lines and mouse tumor therapy model, respectively were investigated. It was the first report of using CYS as a potential drug in cancer therapy. It showed potent significant inhibitory effects on proliferation, migration, and invasion of all tumor cell lines in vitro. It also in vivo inhibited tumor growth and improved mice survival. The investigators attributed their results to the effects of RhCyst-1 in decreasing the number of myeloidderived suppressor cells (MDSCs) from the peripheral blood mononuclear cells (PBMCs). However, the investigators recommended further studies to discover the link between RhCyst-1 and MDSCs regulation from the spleens and PBMCs ${ }^{(60)}$.

As regards $I$. ovatus, a vector transmitting viral encephalitis in Japan, Parizi et al., ${ }^{(61)}$ succeeded to identify CYS type II (five variants, JpIoCys2a-e). Their expression differs among various developmental stages and tissues (e.g. salivary gland, gut, ovaries, and fat bodies), and showed variable inhibitory efficacy against the proteolytic activity of Caths B, C, and L. The investigators also showed their essential role during modulation of initial hemoglobin degradation in blood feeding process through its significant variable inhibitory activity against different Caths. In silico and in vitro comparative cross-antigenicity analyses of JpIoCys2 and two CYS isolated from Rhipicephalus microplus, a cattle ectoparasite with veterinary importance, showed high cross-antigenicity between native and recombinant CYSs. Based on the established development of a vaccine utilizing $R m$ Cys-2 in immunization of cattle for livestock control, the investigators suggested a similar vaccine to control transmission of viral encephalitis in Japan. Similarly, another three variants of CYS2 were recently described and successfully sequenced from Japanese tick transmitting viral encephalitis in Japan, I. persulcatus (JpIpCys2a-c). Their structural analysis and expression profile were demonstrated, and the investigators showed that their transcripts, except for JpIpCys2c, were detected in almost all tissues of all the developmental stages. They noticed the absence of the latter transcripts in unfed larvae $^{(62)}$.

\section{Concluding remarks}

- Salivary glands in all BFAs secrete CPs, mainly Caths, essentially for hemoglobin degradation prior to egg production. Three other roles were assigned; evasion of host defense response, production of inflammatory reactions, and embryogenesis.
- $\quad$ Caspase is the second expressed CP which has an essential role in apoptosis of elements involved in vector's innate immune response against intracellular transmitting microorganisms.

- Use of specific CPIs would be a new strategy for vector control instead of routine insecticides.

- Group 1 allergen (Der-1), the main antigenic element of house dust mites, exhibits CP proteolytic activities, and proved to have a major role in pathogenesis of atopic dermatitis as well as respiratory asthmatic attacks.

- Sequence analysis of gene(s) encoding CPs in scabies mites, identified five SMIPP-Cs homologs with close similarity to Der-1 allergens. They are intensely localized in the intestinal tract and feces, indicating their gut origin, of all burrowing stages with the highest in female mites. Further studies are recommended to assign their exclusive role in interaction with host epidermal tissue and host immune response.

- Ticks CYSs, as components of salivary glands, attract much attention in several publications. The most common identified and characterized CYSs belong to type II (sialostatins), that are essentially involved in impairment of the functions of host immune response through their roles in interference with intracellular and extracellular pathways leading to inhibition of hemostasis.

- Due to the modulatory inhibitory effects on Caths, ticks CYSs are considered potential targets for development of novel drugs and vaccine candidates for several human-tick-borne diseases such as Lyme disease and encephalitis. In addition, several studies recommended use of tick CYSs in cancer therapy, neurodegenerative disorders as well as psoriasis, muscular dystrophy, osteoporosis and rheumatoid arthritis.

Conflict of interest: There is no conflict of interest.

Financial support and sponsorship: Nil.

\section{REFERENCES}

1. Sojka D, Francischetti IBM, Calvo E, Kotsyfakis M. Cysteine proteases from blood feeding arthropod ectoparasites. Adv Exp Med Biol 2011; 712: 177-191.

2. Fouda MA, Hassan MI, Hammad KM, Hasaballah AI. Effects of midgut bacteria and two protease inhibitors on the reproductive potential and midgut enzymes of Culex pipiens infected with Wuchereria bancrofti. J Egypt Soc Parasitol 2013; 43(2):537546. 
3. Cho WL, Tsao SM, Hays AR Walter R, Chen JS, Snigirevskaya ES, et al. Mosquito cathepsin B-like protease involved in embryonic degradation of vitellin is produced as a latent extraovarian precursor. J Biol Chem 1999; 274:13311-13321.

4. Uchida K, Ohmori D, Ueno T, Nishizuka M, Eshita Y, Fukunaga A, et al. Preoviposition activation of cathepsin-like proteinases in degenerating ovarian follicles of the mosquito Culex pipiens pallens. Dev Biol 2001; 237:68-78.

5. Uchida K, Nishizuka M, Ohmori D, Ueno T, Eshita Y, Fukunaga A. Follicular epithelial cell apoptosis of atretic follicles within developing ovaries of the mosquito Culex pipiens pallens. J Insect Physiol 2004; 50(10):903-912.

6. Hurd H, Grant KM, Arambage SC. Apoptosislike death as a feature of malaria infection in mosquitoes. Parasitology 2006;132 Suppl:S33-47.

7. Moura AS, Cardoso AF, Costa-da-Silva AL, Winter CE, Bijovsky AT. Two cathepsins B are responsible for the yolk protein hydrolysis in Culex quinquefasciatus. PLoS One 2015; 10(2): e0118736.

8. Cooper DM, Pio F, Thi EP, Theilmann D, Lowenberger C. Characterization of Aedes Dredd: a novel initiator caspase from the yellow fever mosquito, Aedes aegypti. Insect Biochem Mol Biol 2007; 37(6):559-569.

9. Yan J, Cheng Q Li CB, Aksoy S. Molecular characterization of three gut genes from Glossina morsitans morsitans: Cathepsin B, zincmetalloprotease and zinc-carboxypeptidase. Insect Mol Biol 2002, 11:57-65.

10. Ruttayaporn N, Zhou M, Suganuma K, Yamasaki S, Kawazu S, Inoue N. Expression and characterization of cathepsin B from tsetse (Glossina morsitans morsitans). Jpn J Vet Res 2013; 61(4):137-147.

11. Billingsley PF, Downe AER. Ultrastructural localisation of cathepsin $B$ in the midgut of Rhodnius prolixus Stål (Hemiptera: Reduviidae) during blood digestion. Int J Insect Morph Embryol 1988; 17:295-302.

12. Kollien AH, Waniek PJ, Nisbet AJ, Billingsley PF, Schaub GA. Activity and sequence characterization of two cysteine proteases in the digestive tract of the reduviid bug Triatoma infestans. Insect Mol Biol 2004, 13:569-579.

13. Ferreira-DaSilva CT, Gombarovits ME, Masuda $H$ Oliveira CM, Carlini CR. Proteolytic activation of canatoxin, a plant toxic protein, by insect cathepsinlike enzymes. Arch Insect Biochem Physiol 2000; 44:162-171.
14. Chua KY, Stewart GA, Thomas WR, Simpson RJ, Dilworth RJ, Plozza TM, et al. Sequence analysis of cDNA coding for a major house dust mite allergen, Der $p 1$ homology with cysteine proteases. J Exp Med 1988; $167: 175-182$.

15. Topham CM, Srinivasan N, Thorpe CJ, Overington JP, Kalsheker NA. Comparative modelling of major house dust allergen $\operatorname{Der} p$ 1: structure validation using an extended environmental amino acid propensity table. Protein Eng 1994; 7:869-894.

16. Shakib F, Schulz 0, Sewell HF. A mite subversive cleavage of CD23 and CD25 by Der $p 1$ enhances allergenicity. Immunol Today 1998; 19, 313-316.

17. Gough L, Schulz O, Sewell HF, Shakib F. The cysteine protease activity of the major dust mite allergen Der $p 1$ selectively enhances the immunoglobulin E antibody response. J Exp Med 1999; 190:1897-1902.

18. Kato T, Takai T, Mitsuishi $\mathrm{K}$, Okumura $\mathrm{K}$, Ogawa $\mathrm{H}$. Cystatin A inhibits IL-8 production by keratinocytes stimulated with $\operatorname{Der} p 1$ and $\operatorname{Der} f 1$ : biochemical skin barrier against mite cysteine proteases. J Allergy Clin Immunol 2005; 116(1):169-176.

19. John RJ, Rusznak C, Ramjee $M$, Lamont AG, Abrahamson M, Hewitt EL. Functional effects of the inhibition of the cysteine protease activity of the major house dust mite allergen $\operatorname{Der} p 1$ by a novel peptide-based inhibitor. Clin Exp Allergy 2000; 30(6):784-793.

20. Vasilopoulos Y, Cork MJ, Teare D, Marinou I, Ward SJ, Duff GW, et al. A nonsynonymous substitution of cystatin A, a cysteine protease inhibitor of house dust mite protease, leads to decreased mRNA stability and shows a significant association with atopic dermatitis. Allergy 2007; 62(5):514-519.

21. Ogawa T, Takai T, Kato T, Kikuchi Y, Niyonsaba F, Ikeda $\mathrm{S}$, et al. Upregulation of the release of granulocytemacrophage-colony-stimulating factor from keratinocytes stimulated with cysteine protease activity of recombinant major mite allergens, Der $f 1$ and Der $p$ 1. Int Arch Allergy Immunol 2008; 146(1):27-35

22. Ghaemmaghami AM, Shakib F. Human T cells that have been conditioned by the proteolytic activity of the major dust mite allergen Der $p 1$ trigger enhanced immunoglobulin E synthesis by B cells. Clin Exp Allergy 2002; 32:728-732.

23. Brown A, Farmer K, MacDonald L, Kalsheker N, Pritchard D, Haslett C, et al. House dust mite Der $p 1$ downregulates defenses of the lung by inactivating elastase inhibitors. Am J Respir Cell Mol 2003; 29:381-389. 
24. Deb R, Shakib F, Reid K, Clark H. Major house dust mite allergens Dermatophagoides pteronyssinus 1 and Dermatophagoides farinae 1 degrade and inactivate lung surfactant proteins A and D. J Biol Chem 2007; 282:36808-36819.

25. Newton GK, Perrior TR, Jenkins K, Major MR, Key $\mathrm{RE}$, Stewart MR, et al. The discovery of potent, selective, and reversible inhibitors of the house dust mite peptidase allergen $\operatorname{Der} p$ 1: an innovative approach to the treatment of allergic asthma. J Med Chem 2014; 57(22):9447-9462.

26. Reddy VB, Lerner EA. Activation of mas-related G-protein-coupled receptors by the house dust mite cysteine protease $\operatorname{Der} p 1$ provides a new mechanism linking allergy and inflammation. J Biol Chem 2017; 292(42):17399-17406.

27. Thomas WR. Hierarchy and molecular properties of house dust mite allergens. Allergol Int 2015; 64:304-311.

28. Randall TA, London RE, Fitzgerald MC, Mueller GA. Proteases of Dermatophagoides pteronyssinus. Int J Mol Sci 2017; 18(6): 1204.

29. Holt DC, Fischer K, Pizzutto SJ, Currie BJ, Walton SF, Kemp DJ. A multigene family of inactivated cysteine proteases in Sarcoptes scabiei. J Invest Dermatol 2004; 123:240-241.

30. Lee AJ, Machell J, Van Den BroekAH, Nisbet AJ, Miller $\mathrm{HR}$, Isaac RE, et al. Identification of an antigen from the sheep scab mite, Psoroptes ovis, homologous with house dust mite group I allergens. Parasite Immunol 2002; 24:413-422.

31. Fernando DD, Reynolds SL, Zakrzewski M, Mofiz E, Papenfuss AT, Holt DC, et al. Phylogenetic relationships, stage-specific expression and localization of a unique family of inactive cysteine proteases in Sarcoptes scabiei. Parasit Vectors 2018; 11(1):301.

32. Schwarz A, Valdés JJ, Kotsyfakis M. The role of cystatins in tick physiology and blood feeding ticks. Tick Borne Dis; 2012, 3(3):117-127.

33. Chmelař J, Kotál J, Langhansová H, Kotsyfakis M. Protease inhibitors in tick saliva: the role of serpins and cystatins in tick-host-pathogen interaction. Front Cell Infect Microbiol 2017; 7:216.

34. Valdés-Rodríguez S, Galván-Ramírez JP, GuerreroRangel A, Cedro-Tanda A. Multifunctional amaranth cystatin inhibits endogenous and digestive insect cysteine endopeptidases: A potential tool to prevent proteolysis and for the control of insect pests. Biotechnol Appl Biochem 2015; 62(5):634-841.
35. Anderson JM, Sonenshine DE, Valenzuela JG. Exploring the mialome of ticks: an annotated catalogue of midgut transcripts from the hard tick, Dermacentor variabilis (Acari: Ixodidae). BMC Genomics 2008; 9:552.

36. Sojka D, Hajdušek 0 , Dvořák J, Sajid M, Franta $\mathrm{Z}$, Schneider EL, et al. IrAE: An asparaginyl endopeptidase (legumain) in the gut of the hard tick Ixodes ricinus. Int J Parasitol 2007; 37:713-724.

37. Sojka D, Franta Z, Horn M, Hajdusek O, Caffrey CR, Mares M, et al. Profiling of proteolytic enzymes in the gut of the tick Ixodes ricinus reveals an evolutionarily conserved network of aspartic and cysteine peptidases. Parasit Vectors; 2008, 1:7.

38. Abdul-Alim M, Tsuji N, Miyoshi T, Khyrul-Islam $\mathrm{M}$, Huang X, Motobu M, et al. Characterization of asparaginyl endopeptidase, legumain induced by blood feeding in the ixodid tick Haemaphysalis longicornis. Insect Biochem Mol Biol 2007; 37(9):911-922.

39. Abdul-Alim M, Tsuji N, Miyoshi T, Islam MK, Hatta T, Fujisaki K. Legumains from the hard tick Haemaphysalis longicornis play modulatory roles in blood feeding and gut cellular remodelling and impact on embryogenesis. Int J Parasitol 2009; 39(1):97-107.

40. Tsuji N, Miyoshi T, Battsetseg B Matsuo T, Xuan X, Kozo Fujisaki K. A cysteine protease is critical for Babesia spp. transmission in Haemaphysalis ticks. PLoS Pathog 2008; 4:e1000062.

41. Yamaji K, Tsuji N, Miyoshi T Islam MK, Hatta T, Alim MA et al. Hemoglobinase activity of a cysteine protease from the ixodid tick Haemaphysalis longicornis. Parasitol Int 2009; 58:232-237.

42. Estrela A, Seixas A, Termignoni C. A cysteine endopeptidase from tick Rhipicephalus (Boophilus) microplus larvae with vitellin digestion activity. Comp Biochem Physiol B Biochem Mol Biol 2007; 148:410-416.

43. Yu X, Gong H, Zhou Y, Zhang H, Cao J, Zhou J. Differential sialotranscriptomes of unfed and fed Rhipicephalus haemaphysaloides, with particular regard to differentially expressed genes of cysteine proteases. Parasit Vectors 2015; 8:597.

44. Yu X, Zhou Y, Cao J, Zhang H, Gong H, Zhou J. Caspase-1 participates in apoptosis of salivary glands in Rhipicephalus haemaphysaloides. Parasit Vectors 2017; 10(1):225.

45. Valenzuela JG, Francischetti IM, Pham VM, Garfield MK, Mather TN, Ribeiro JM. Exploring the sialome 
of the tick Ixodes scapularis. J Exp Biol 2002; 205(18):2843-2864.

46. Kotsyfakis M, Sá-Nunes A, Francischetti IM, Mather TN, Andersen JF, Ribeiro JM. Anti-inflammatory and immunosuppressive activity of sialostatin $\mathrm{L}$, a salivary cystatin from the tick Ixodes scapularis. J Biol Chem 2006; 281(36):26298-26307.

47. Kotsyfakis M, Karim S, Andersen JF, Mather TN, Ribeiro JM. Selective cysteine protease inhibition contributes to blood-feeding success of the tick Ixodes scapularis. J Biol Chem 2007; 282(40):29256229263.

48. Lieskovská J, Palenikova J, Langhansova $\mathrm{H}$, Campos Chagas A, Calvo E, Kotsyfakis $\mathrm{M}$, et al. Tick sialostatins L and L2 differentially influence dendritic cell responses to Borrelia spirochetes. Parasit Vectors 2015: 8:275.

49. Lieskovská J, Páleníková J, Širmarová J, Elsterová J, Kotsyfakis M, Campos Chagas A, et al. Tick salivary cystatin sialostatin L2 suppresses IFN responses in mouse dendritic cells. Parasite Immunol 2015; 37(2):70-78.

50. Karim S, Miller NJ, Valenzuela JG, Sauer JR, Mather TN. RNAi-mediated gene silencing to assess the role of synaptobrevin and cystatin in tick blood feeding. Biochem Biophys Res Commun 2005; 334:1336-1342.

51. Salát J, Paesen GC, Rezácová P, Kotsyfakis M, Kovárová Z, Sanda $\mathrm{M}$ et al. Crystal structure and functional characterization of an immunomodulatory salivary cystatin from the soft tick Ornithodoros moubata. Biochem J 2010; 429(1):103-112.

52. Ribeiro JM, Franischetti IM. Role of arthropod saliva in blood feeding: sialome and post-sialome perspectives. Annu Rev Entomol 2003; 48:73-88.

53. Horka H, Staudt V, Klein M, Taube C, Reuter S, Dehzad $\mathrm{N}$ et al. The tick salivary protein sialostatin $\mathrm{L}$ inhibits the Th9-derived production of the asthma-promoting cytokine IL-9 and is effective in the prevention of experimental asthma. J Immunol 2012; 188(6):2669-2676.
54. Grunclová L, Horn M, Vancová M, Sojka D, Franta Z, Mares $\mathrm{M}$, et al. Two secreted cystatins of the soft tick Ornithodoros moubata: differential expression pattern and inhibitory specificity. Biol Chem 2006; 387(12):1635-1644.

55. Yamaji K, Tsuji N, Miyoshi T, Islam MK, Hatta T, Alim MA, et al. A salivary cystatin, HISC-1, from the ixodid tick Haemaphysalis longicornis play roles in the blood-feeding processes. Parasitol Res 2009; 106(1):61-68.

56. Yamaji K, Tsuji N, Miyoshi T, Hatta T, Alim MA, Anisuzzaman, et al. Hlcyst-1 and Hlcyst-2 are potential inhibitors of HICPL-A in the midgut of the ixodid tick Haemaphysalis longicornis. J Vet Med Sci 2010; 72(5):599-604.

57. Zhou J, Liao $M$, Gong $H$, Xuan $X$, Fujisaki K. Characterization of Hlcyst-3 as a member of cystatins from the tick Haemaphysalis longicornis. Exp Appl Acarol 2010; 51(4):327-333.

58. Wang Y, Yu X, Cao J, Zhou Y, Gong H, Zhang H, et al. Characterization of a secreted cystatin from the tick Rhipicephalus haemaphysaloides. Exp Appl Acarol 2015; 67(2):289-298.

59. Wang Y, Zhou Y, Gong H, Cao J, Zhang H, Li X, Zhou J. Functional characterization of a cystatin from the tick Rhipicephalus haemaphysaloides. Parasit Vectors 2015; 8:140.

60. Wei N, Lin Z, Xu Z, Cao J, Zhou Y, Zhang H, et al. A tick cysteine protease inhibitor RhCyst-1 exhibits antitumor potential. Cell Physiol Biochem 2018; 46(6):2385-2400.

61. Parizi LF, Sabadin GA, Alzugaray MF, Seixas A, Logullo C, Konnai S, et al. Rhipicephalus microplus and Ixodes ovatus cystatins in tick blood digestion and evasion of host immune response. Parasit Vectors 2015; 8:122.

62. Rangel CK, Parizi LF, Sabadin GA, Costa EP, Romeiro NC, Isezaki $\mathrm{M}$, et al. Molecular and structural characterization of novel cystatins from the taiga tick Ixodes persulcatus. Ticks Tick Borne Dis 2017; 8:432-441. 